\title{
Rational Number
}

National Cancer Institute

\section{Source}

National Cancer Institute. Rational Number. NCI Thesaurus. Code C62441.

A number that can be expressed as a fraction $p / q$ where $p$ and $q$ are integers and $q$ does not equal zero. 